\title{
A COMPACT FOURTH-ORDER MULTI-FOLD HAIRPIN LINE MICROSTRIP BANDPASS FILTER AT 1650 MHZ FOR RF/ WIRELESS COMMUNICATIONS
}

\author{
Jagdish Shivhare ${ }^{1}$, S B Jain ${ }^{2}$ \\ ${ }^{1}$ Department of Electrical, Electronics and Communication Engineering, ITM University, Sector-23A, Gurgaon-122 \\ 017, India \\ ${ }^{2}$ Department of Electronics and Communication, Indira Gandhi Institute of Technology, Indraprashth University, \\ Delhi, India
}

\begin{abstract}
This paper describes the design, simulation/optimization and measured results of a fourth-order multi-folded hairpin line microstrip bandpass filter at $1650 \mathrm{MHz}$, with 60-65\% reduction in size compared to the structure of a basic hairpin line microstrip bandpass filter. The imperical equations and relevant graphs have been used to realize adjacent and cross couplings between the four multi-folded resonators. A conventional hairpin-line resonator size is normally very large. The folded hairpin line resonator filters are compact, small sized and simple to design and fabricate. The proposed multi-folded hairpin line microstrip filter is a slow wave open loop type narrow band, high selectivity, small sized and low cost band pass filter for RF/wireless trans/receive communication systems for ground and space applications in L-band. The cross couplings have been realized between adjacent and non-adjacent resonators. The ADS-Agilent make software have been used to design and simulate the filters having the folded hairpin line resonators. The measured results are very close to the simulated results, with great reduction in size compared to the conventional planar hairpin line structure.
\end{abstract}

Keywords-Hairpin line, Centre frequency, frequency response cross couplings, Multi-fold resonator, pass-band bandwidth, attenuation band, adjacent resonators

\section{INTRODUCTION}

Filters using multi-fold miniaturized resonators are $60-65 \%$ smaller in size compare to the filters that use conventional hairpin line microstrip resonators. The hairpin line resonators are composed of a transmission line and a lumped element capacitor and is represented by parallel-coupled lines instead of the lumped capacitor, shown in fig.1a,1b, 1c, 1d. and 1.e. The design technique uses an approximation polynomial and a low filter prototype. The loaded $\mathrm{Q}$ factor and the mixed coupling coefficients between the different resonators can be calculated by using imperical equations[1]-[4]. The simulation/optimization work has been carried out by using ADS, Agilent-make software.
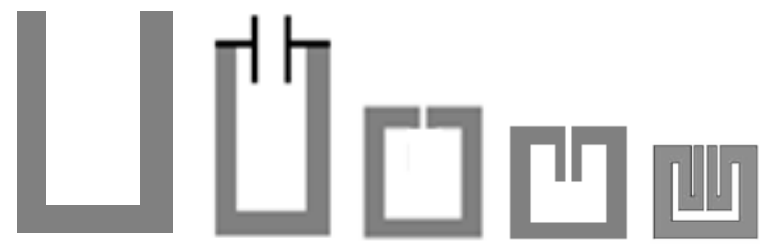

Fig.1-a Fig.1-b Fig.1-c Fig.1-d Fig.1-e
In the proposed filter the multi-folded resonators are cross coupled. Such a filter can be realized by using cross coupling between adjacent resonators[3]-[8].We have used imperical equations and relavent graphs to calculate the total size of the fourth-order conventional hairpin line, singlefold, double-fold and Multi-fold hairpin line bandpass filters and their overall dimensions of are $400 \mathrm{~mm}^{2}$ (A), $260 \mathrm{~mm}^{2}$ (65\% of A), $200 \mathrm{~mm}^{2}$ (50\% of A) $144 \mathrm{~mm}^{2}$ (36\% of A) respectively.

\section{THEORY}

The folded filters are shown in figures 2,3 and 4 . The proposed multi-fold hairpin line microstrip bandpass filter is shown in fig.4.The filter has fourth-order cross-coupled structure. In this configuration, significant couplings exist between any two adjacent resonators. The capacitive couplings between resonators 1 and 3 and resonators 2 and 4 are negligible.

Fig 1 Actual, capacitor loaded, single-fold, double-fold and multi-fold hairpin line microstrip resonators 


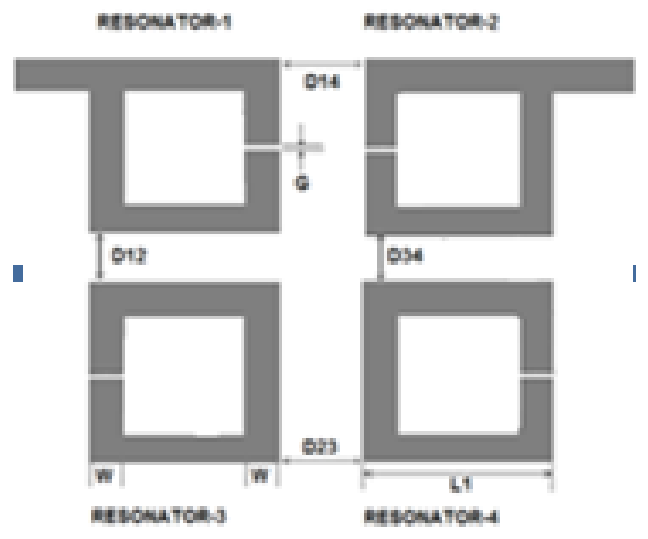

Fig 2 Fourth-order single-fold bandpass filter of $260 \mathrm{~mm}^{2}$

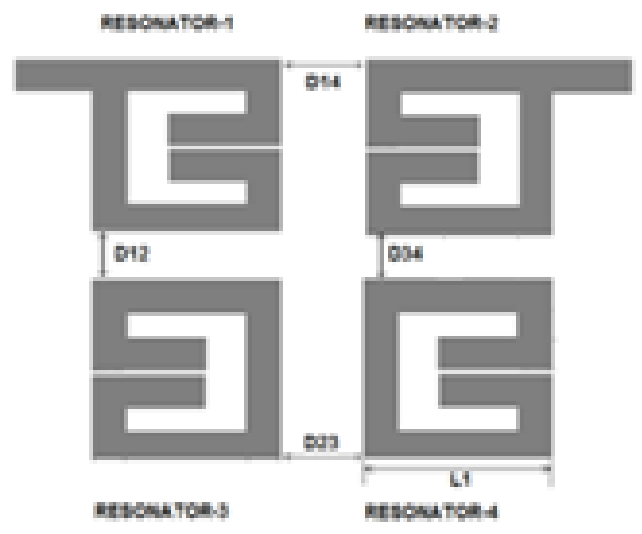

Fig.3 Fourth-order double-fold bandpass filter of $200 \mathrm{~mm}^{2}$

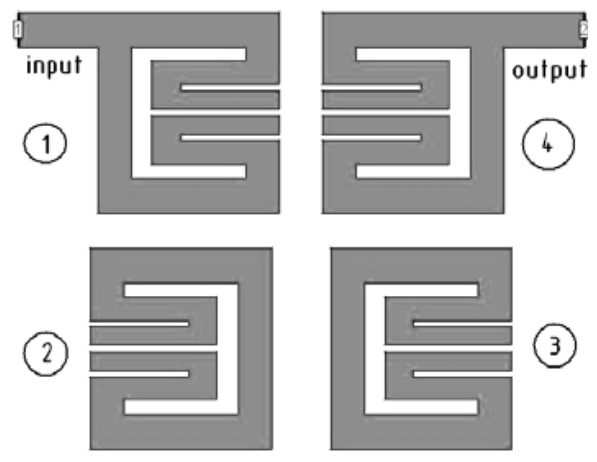

Fig.4 Fourth-order multi-fold bandpass filter of $170 \mathrm{~mm}^{2}$

\section{DESIGN PROCEDURE}

To reduce the size of hairpin resonators, the arms of the resonator are folded to reduce the size compare to the conventional hairpin resonators[9]-[16]. Design calculations of a fourth-order multi-folded filter can be done in the following steps:

1. Finding the element values of LPF prototype by using the approximate synthesis method.The relations between the bandpass design parameters and the lowpass elements [1-2] are:

$$
\begin{aligned}
& Q_{e i}=Q_{e}=C_{1} / \Delta \omega \\
& k_{n, n-1}=k_{N-n, N-n+1}=\frac{\Delta \omega}{\sqrt{C_{n} C_{n+1}}} \text {, for } n=1 \text { to } N / 2 \\
& k_{m, m+1}=\frac{\Delta \omega J_{m}}{C_{m}}, \text { for } m=N / 2, \\
& k_{m-1, m+2}=\frac{\Delta \omega J_{m-1}}{C_{m-1}}, \text { for } m=N / 2,
\end{aligned}
$$

Where ${ }^{\Delta \omega}$ : fractional bandwidth of the bandpass filter, $\mathrm{C}$ : Capacitance of the lumped capacitor $\mathrm{J}$ : Characteristric admittance of the inverter, $\mathrm{N}$ : degree of the filter

2. To calculate the resonator parameters: The length of the coupled lines can be calculated by:

$$
\begin{aligned}
& \cot g \theta_{p}=\frac{-R+\sqrt{R^{2}+4 Z_{c}^{2} \sin ^{2} \theta_{s}}}{2 Z_{c} \sin \theta_{s}} \\
& \text { and } R=\left(Z_{p e}+Z_{p o}\right) \cos \theta_{s}-\left(Z_{p e}-Z_{p o}\right) .
\end{aligned}
$$

Where $\theta_{s}$ : Electric length of the resonator $Z_{c}$ : Characteristic impedance $Z_{p e}$ :Even mode impedance $Z_{p o}$ : Odd mode impedance

3. Calculations for the coupling parameters: The values of coefficient of coupling between resonators can be calculated against the distances between the resonators. The design technique uses an approximation polynomial and a low filter prototype. The loaded $\mathrm{Q}$ factor and the mixed coupling coefficients between different resonators can be calculated by using the equations, graphs and commercial softwares.

4. Calculation of the input tapped electrical length:

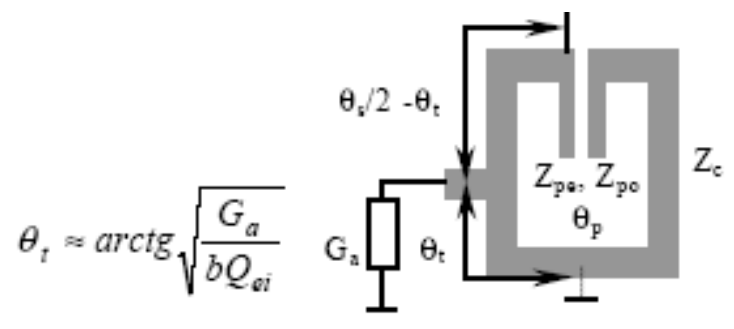

Fig.5 Input/output tapped electrical length

5. Calculation of the geometric parameters of the filter for an exact substrate.

6. Optimization of filter parameters by varying the geometric dimensions. In this work we have used Alumina substrate of dielectric constant of 10.2, thickness of 1.27 $\mathrm{mm}$ and tangent loss of 0.003 


\section{FILTER DESIGN SPECIFICATIONS}

1. Center Frequency: $1650 \mathrm{MHz}$

2. Insertion Loss: $<3 \mathrm{~dB}$

3. $3 \mathrm{~dB}$ Bandwidth: $\pm 20 \mathrm{MHz}$ w. r. t. c. f

4. $30 \mathrm{~dB}$ Bandwidth: $\pm 50 \mathrm{MHz}$ w. r. t. c. $\mathrm{f}$

5. $40 \mathrm{~dB}$ Bandwidth: $\pm 80 \mathrm{MHz}$ w. r. t. c. f

6. Input impedance: $50 \mathrm{Ohms}$

7. Output impedance: $50 \mathrm{Ohms}$

\section{CALCULATED DIMENSIONS OF THE}

\section{FILTER}

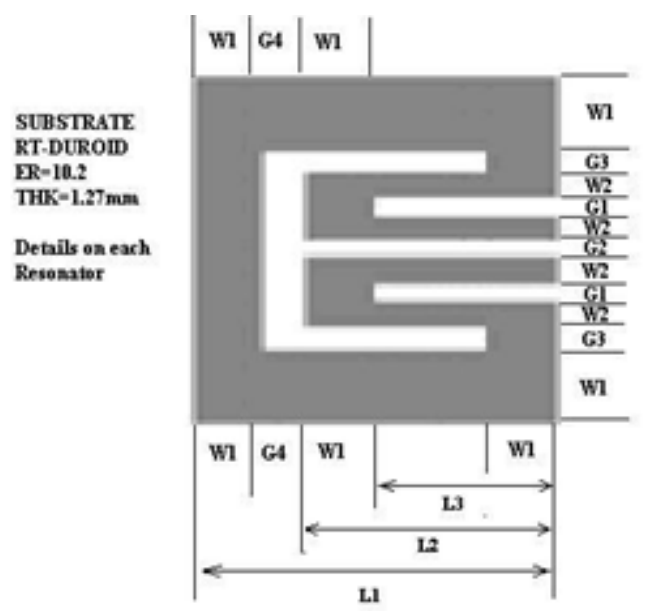

Fig 6 Dimensions of a multi-fold hairpin line resonator

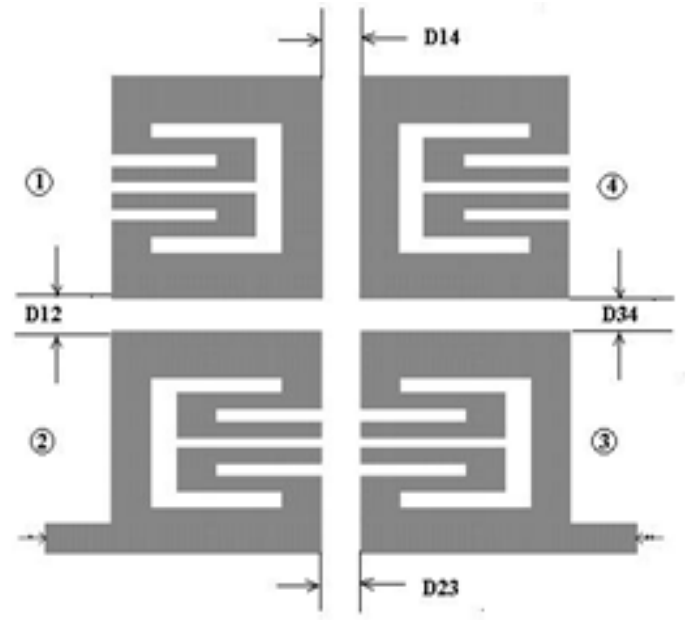

Fig 7 Arrangement of four resonators in the filter

Here, $\mathrm{L}_{1}: 5.35 \mathrm{~mm}, \mathrm{~L}_{2}: 3.23 \mathrm{~mm}, \mathrm{~L} 3: 1.89 \mathrm{~mm}$ $\mathrm{W} 1: 1.34 \mathrm{~mm} \mathrm{~W} 2: 0.53 \mathrm{~mm}, \mathrm{G} 1: 0.44 \mathrm{~mm}$ G2 : $0.39 \mathrm{~mm} \mathrm{G} 3: 0.43 \mathrm{~mm}, \mathrm{G} 4: 0.77 \mathrm{~mm}$ $\mathrm{D}_{12}: 1.3 \mathrm{~mm}=\mathrm{D} 34: 1.31 \mathrm{~mm}, \mathrm{D}_{23}=\mathrm{D} 14: 1.21 \mathrm{~mm}$

* Total size: $12 \mathrm{~mm}$ x $12 \mathrm{~mm}\left(144 \mathrm{~mm}^{2}\right.$ i.e. $36 \%$ of A)

Where A: $400 \mathrm{~mm}^{2}$, is the total size of the conventional hairpin line microstrip bandpass filter at center frequency $1650 \mathrm{MHz}$

\section{SIMULATED AND MEASURED RESULTS:}

Simulated results by using ADS Agilent-make software:

1. Center Frequency: $1649 \mathrm{MHz}$

2. Insertion Loss: < $3 \mathrm{~dB}$

3. 3dB Bandwidth: $\pm 19 \mathrm{MHz}$ w. r. t. c. f

4. $30 \mathrm{~dB}$ Bandwidth: $\pm 51 \mathrm{MHz}$ w. r. t. c. f

5. $40 \mathrm{~dB}$ Bandwidth: $\pm 78 \mathrm{MHz}$ w. r. t. c. $\mathrm{f}$

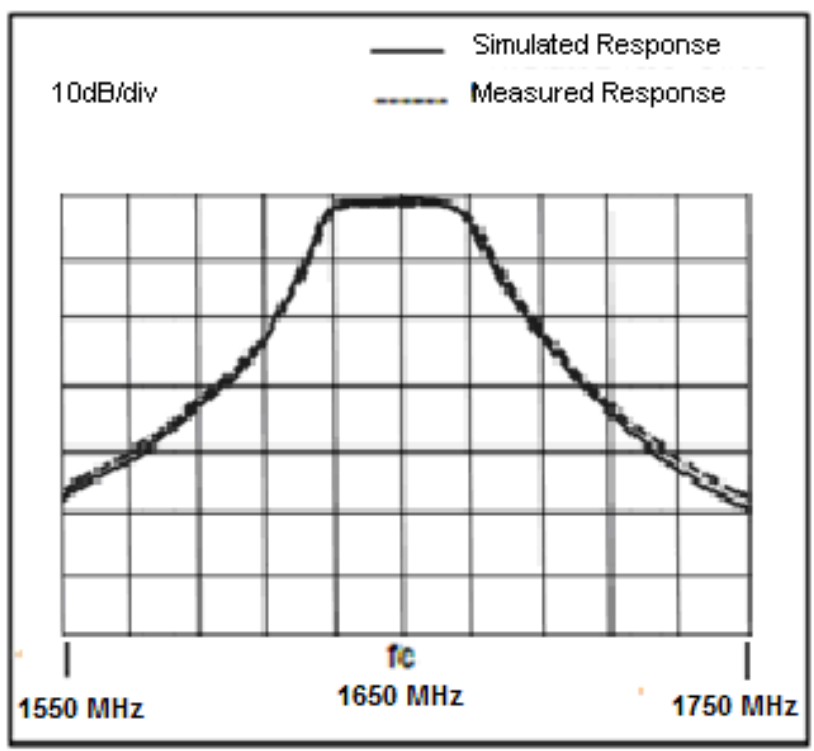

Fig 8 Simulated and measured responses of multifold hairpin line microstrip bandpass filter at $1650 \mathrm{MHz}$

Measured results on Network Analyzer:

1. Center Frequency: $1648 \mathrm{MHz}$

2. Insertion Loss: $<3 \mathrm{~dB}$

3. $3 \mathrm{~dB}$ Bandwidth: $\pm 18 \mathrm{MHz}$ w. r. t. c. f

4. $30 \mathrm{~dB}$ Bandwidth: $\pm 52 \mathrm{MHz}$ w. r. t. c. f

5. $40 \mathrm{~dB}$ Bandwidth: $\pm 78 \mathrm{MHz}$ w. r. t. c. $\mathrm{f}$

\section{FABRICATION OF FILTER}

The filter circuit is fabricated on Alumina substrate having dielectric constant, er:10.2 and thickness $1.27 \mathrm{~mm}$. Standard fabrication process have been adopted. The measured results are very close to the simulated results and designed specifications.

\section{CONCLUSIONS}

This paper presents a fourth-order multi-fold hairpin line microstrip bandpass filter design. The simulation/optimization work has been carried out by using ADS Agilent-make software. The limitations of the design calculation/simulation results in terms of inaccuracy of capacitive and inductive coupling between the adjacent and cross coupled multi-fold hairpin line microstrip resonators. The measured results are very close to the simulated results and the overall size of the multi-fold filter is approximately $40 \%$ of the size of the conventional hairpin line filter i.e. the size is reduced by $60 \%$. 


\section{REFERENCES}

[1]. J. S. Hong and M J Lancaster, Microstrip filters for RF/Microwave Applications, New York: Wiely, 2001

[2]. M. Sagawa, K. Takahashi and M. Makimoto "Miniaturized hairpin resonator filters and their applications to receiver front-end MIC's, IEEE Trans. MTT37,1989,p.1991-1997.

[3]. Hong,J. S. and M. J. Lancaster, “ Couplings Microstrip Square Open-Loop Resonators for Cross-Coupled Planar Microstrip Filters" IEEE Trans.Microw.Theory Tech. Vol. No. 5, October 2006.

[4]. Ilia G. Iliev and Marin V Nedelchev "CAD of Cross Coupled Miniaturized Hairpin Bandpass Filters" Microwave Review ,pp-49-52,December 2002.

[5]. Rodrigo Neves Martins and Humberto Abdalla Jr."Techniques Yield Tiny Hairpin-Line Resonator Filters" MWRF, pp 1-11, November 1999.

[6]. Deng P H,Y S Lin,C H Wang and C H Chen,"Compact Microstrip bandpass filters with good selectivity and Stopband rejection" IEEE Trasanction on Microwave Theory and Te- chniques, Vol.54, No.2, pp533-39, 2006.

[7]. Sarawuth Chaimool,Sithiporn Kerdsumang and Prayoot Akkaraekthalin "Design of an Improved Four-Pole Hairpin Resonator Filter,The Journal of KMITNB,Vol.15, No. 2, pp 07-12, April-June 2005,

[8]. Wang Y X,B-Z Wang and J Wang "A compact square loop dual mode bandpass filter with wide stopband" Progress in Electromagnetics Research,Vol.77,pp 67-73, 2007

[9]. Jen-Tsai Kuo, Ming-Jyh Maa and Ping-Han Lu "A Micro- strip Elliptic Function Filter with Compact Miniaturized Hairpin Resonators" IEEE Microwave and Guided Wave Letters, Vol 10, No.3, pp-94-95, March 2000.

[10]. Siniša Jovanović, Aleksandar Nešić: "New Filter Type Suitable for Miniature Printed Bandpass Filters at RF \& Microwave Frequencies" European Microwave Conference, Proceedings of Papers pp. 25-28 Paris, 3-7 Oct. 2005.

[11]. Siniša Jovanović, Aleksandar Nešić: "Printed BandPass Filter at L Band", 14th IST Mobile \& Wireless Communi- cations Summit, Dresden, 19-23 June 2005.

[12]. S. Jovanović, A. Nešić: "Microstrip bandpass filter with new type of capacitive coupled resonators", Electronics Letters, Vol.41, No. 1, pp 19-21, January 2005.

[13]. Tsai, W.L. and R.B. Wu. "Tri-band Filter design using substrate integrated waveguide resonators in LTCC. Proceeding of the IEEE International Symposium on MTT-S Microwave Digest. 2011.

[14]. Fei, L., H. Gan, Z. Wang and W. Lu, “ Novel Compact triple-bandpass filter using $\lambda / 4$ resonator pairs with common via ground" Proceeding of the Progress in Electromagnetics Research Symposium, pp: 1220-24, 2012

[15]. Lakhan Singh, Dr. P.K. Singhal, "Design and Analysis of Hairpin Line Bandpass Filter" International Journal of Advanced Research in Electronics and Communication Engineering (IJARECE), Volume 2, Issue 2, February 2013(ISSN: $2278-909 X)$

[16]. ADS Agilent-make Softwares for Design and Simulation 